\title{
A cold change: how short low temperature exposure affects primary metabolism in leaves and stems of two eucalyptus species
}

\author{
Adilson Pereira Domingues-Junior (D) Danilo de Menezes Daloso • \\ Mariana Machado - Laíse Rosado-Souza - Leonardo Perez de Souza • \\ Alisdair Robert Fernie • Paulo Mazzafera
}

Received: 5 February 2019/Accepted: 4 September 2019/Published online: 18 November 2019

(C) The Author(s) 2019, corrected publication 2020

\begin{abstract}
Plants often modify their metabolism in order to regain homeostasis and maintain survival in the face of stressful conditions. Here, two species of eucalyptus, E. globulus and E. grandis (adapted and non-adapted to low temperature, respectively), were exposed to either $10^{\circ} \mathrm{C}$ or $25^{\circ} \mathrm{C}$ over $24 \mathrm{~h}$, and changes in gene expression and metabolite levels were analyzed. The aim of this experiment was to investigate the dynamic of short period changes in the energy metabolism of source (leaves) and sink (stem) tissues
\end{abstract}

Electronic supplementary material The online version of this article (https://doi.org/10.1007/s40626-019-00156-8) contains supplementary material, which is available to authorized users.

A. P. Domingues-Junior · P. Mazzafera

Departamento de Biologia Vegetal, Instituto de Biologia, Universidade Estadual de Campinas, São Paulo, Brazil e-mail: pmazza@unicamp.br

A. P. Domingues-Junior $(\varangle) \cdot$ D. M. Daloso ·

M. Machado - L. Rosado-Souza - L. P. de Souza .

A. R. Fernie

Max-Planck-Institut für Molekulare Pflanzenphysiologie,

Potsdam-Golm, Germany

e-mail: apdominguesjr@usp.br

D. M. Daloso

e-mail: dani.daloso@gmail.com

M. Machado

e-mail: mmachado21@outlook.com

L. Rosado-Souza

e-mail: rosado@mpimp-golm.mpg.de in these contrasting species regarding low temperature. We expected to observe a distinct pattern on carbon metabolism and source-to-sink relationship between both species which would be related to their different vegetative responses when facing low temperatures. In that way, E. globulus plants showed a differential expression in leaves and stems of SnRK1 genes system (responsible for energy availability control in plants), that was strongly associated to the changes in carbon metabolism and the main difference between the response when both species face cold. Taken together, the results suggest that low temperatures $\left(10^{\circ} \mathrm{C}\right)$ are able to increase the sink strength of stem tissues and the carbon assimilation in leaves of $E$.

L. P. de Souza
e-mail: lperez@mpimp-golm.mpg.de

A. R. Fernie

e-mail: fernie@mpimp-golm.mpg.de

D. M. Daloso

Departamento de Bioquímica e Biologia Molecular,

Universidade Federal do Ceará, Fortaleza, Brazil

M. Machado

Instituto de Biociências, Universidade Federal de Goiás Regional Jataí, Jataí, Goiás, Brazil

A. P. Domingues-Junior · P. Mazzafera

Laboratório de Produção Vegetal, Escola Superior de Agricultura "Luiz de Queiroz", Universidade de São Paulo, Piracicaba, São Paulo, Brazil 
globulus, supporting a higher vegetative growth rate. In E. grandis, on the other hand, exposure to $10{ }^{\circ} \mathrm{C}$ promoted a higher consumption of carbon skeletons without better growth rate as a counterpart, suggesting that under cold conditions, these two eucalyptus species differ in the way they coordinate the interaction between the activation of SnRK1 system and primary metabolism in source and sink tissues.

Keywords Eucalyptus - SnRK1 system · Primary metabolism $\cdot$ Cold stress

$\begin{array}{ll}\text { Abbreviations } \\ \text { BCAAs } & \text { Branched-chain amino acids } \\ \text { CDD } & \text { Conserved domains database } \\ \text { CTAB } & \text { Cetyl trimethylammonium bromide } \\ \text { EUCAGEN } & \text { Eucalyptus Genome Network } \\ \text { GC-MS } & \text { Gas chromatography-mass } \\ & \text { spectrometry } \\ \text { GMD } & \text { Golm metabolome database } \\ \text { IDH } & \text { Isocitrate dehydrogenase } \\ \text { KIN10 } & \text { Kinase protein 10 } \\ \text { KIN11 } & \text { Kinase protein 11 } \\ \text { PC } & \text { Principal component } \\ \text { PCA } & \text { Principal components analysis } \\ \text { qRT-PCR } & \text { Quantitative reverse transcription } \\ & \text { polymerase chain reaction } \\ \text { SnRK1 } & \text { SNF-related kinase 1 } \\ \text { TCA } & \text { Tricarboxylic acid cycle } \\ \text { TOR-1 } & \text { Target of rapamycin kinase-1 }\end{array}$

\section{Introduction}

Changes of irradiance and temperature imposed by movements of the Earth are two of the main external events capable of altering plant development (Kooke and Keurentjes 2011; Shin et al. 2017; Tarkowski and Van den Ende 2015). In this sense, although there are differences between later physiological and metabolic responses when plants face abrupt or gradually decreasing temperatures, the molecular pathways firstly activated in response to cold stress are common to both cases, being more sensitive to the range of the minimum temperature achieved (Zarka et al. 2003; Kidokoro et al. 2017; Hao et al. 2018). Cold responses strongly differ if the temperature drops down below ice formation, promoting a freezing stress condition, or if it ranges between 0 and $15{ }^{\circ} \mathrm{C}$, characterized as chilling events (Fenollosa et al. 2018; Hao et al. 2018). Under natural conditions, plants growing in tropical and subtropical zones are more likely to be exposed to chilling events, affecting how they respond to those seasonally events and grow during the year (Fenollosa et al. 2018). Therefore, during autumn and winter seasons, massive changes in gene expression patterns, enzymatic activities, and specific metabolite concentrations tend to slow down plant growth rates, allowing them to decrease energy consumption and survive these harsh periods of the year (O'Brien et al. 2014; Budzinski et al. 2016; Hoermiller Imke et al. 2017). A well-known characteristic of cold-tolerant plants is their ability to continuously provide sugars and other energetic substrates to non-photosynthetic tissues in a minimally altered manner, allowing relatively minor changes in vegetative development during winter (Heidarvand et al. 2017; Hoermiller Imke et al. 2017; O’Brien et al. 2014).

Several other physiological events are regulated by sugar signaling such as those downstream to the Target of rapamycin kinase-1/SNF-related kinase 1 (TOR-1/SnRK1) system. The TOR-1/SnRK1 system is responsible for controlling the availability of nutrients, integrating a wide variety of environmental and endogenous signals and regulating anabolic and catabolic processes in order to maintain energy balance and sustain an optimal growth rate (Caldana et al. 2013; Tomé et al. 2014; Williams et al. 2014; Wurzinger et al. 2018). While the TOR-1 portion of the complex shows high activity in meristematic tissues, the SnRK1 portion is active in both source and sink tissues of plants (Tomé et al. 2014; Williams et al. 2014; Wurzinger et al. 2018). The SnRK1 portion, whose catalytic subunit is composed of the kinase proteins KIN10 and KIN11, is associated with the activation of metabolic processes that increase the amount of available energy, such as higher photosynthetic activity and catabolism, especially in situations of elevated metabolic demand (Baena-Gonzalez et al. 2007; Schluepmann et al. 2011). Additionally, the activity of SnRK1 also depends on the tissue type analyzed. At first, the catabolic behavior promoted by SnRK1 complex may appear to be counter-intuitive in heterotrophic tissues, which require a plenty of substrates available while promote their growth (Schluepmann et al. 2011; Wu and Birch 2010; Wurzinger et al. 2018). Nonetheless, source-sink dynamics must 
be taken into account, in those tissues: when the amount of available sucrose is sufficiently high, SnRK1 activity is inhibited, activating anabolic pathways required for growth. However, as sucrose is used as a substrate for growth, its content falls and thus the activity of SnRK1 increases, facilitating remobilization of stored carbon skeletons to enable the normal growth of these tissues (Bechtold and Field 2018; Halford and Hey 2009; Schluepmann et al. 2011; Wu and Birch 2010). In leaves, due to their high photosynthetic rate, the amount of sucrose tends to be high during the day, inhibiting the activity of SnRK1. However, if a plant is subject to conditions that limit its photosynthesis such as saline and thermal stresses, activation of SnRK1 system and the promotion of pathways favoring a greater carbon fixation are observed (Baena-Gonzalez et al. 2007; Bechtold and Field 2018; Schluepmann et al. 2011).

Cell wall formation represents an important physiological event in plants and carries a high energy burden (Allahverdiyeva et al. 2015; Cerasuolo et al. 2016; Osakabe et al. 2013). In woody plants, cell wall may account for up to $50 \%$ of the total dry mass, mostly represented by the high investment in stem growth (Arias et al. 2014; Campoe et al. 2013; Cerasuolo et al. 2016; Osakabe et al. 2011). Currently, species and hybrids of eucalyptus (genus Eucalyptus, family Myrtaceae) are the main forest crop supplying wood for the cellulose pulp and paper industry (Araújo et al. 2014; Budzinski et al. 2016; Campoe et al. 2013; Turnbull and Booth 2002). E. grandis is amongst the most globally cultivated eucalyptus species and it is valued for its rapid growth, whereas E. globulus is characterized by a better wood quality (related to cellulose yield) and higher productivity compared to $E$. grandis when both species grow under low temperature conditions (Budzinski et al. 2016; Fernández et al. 2010; Ruedell et al. 2013).

The objective of this work was to evaluate the main changes in energy metabolism of E. globulus and $E$. grandis exposed for $24 \mathrm{~h}$ to $10{ }^{\circ} \mathrm{C}$ and identify differential responses in primary metabolism of leaf and stem tissues, representing source and sink photoassimilates tissues, respectively. We expected to identify patterns associated to each tissue and how they change under low temperature, and the impact on the energy availability according to catabolic and anabolic regulations. Therefore, we could observe that the differential stem growth of E. globulus compared to E. grandis when both species grow under cold environments (Domingues-Junior et al., MS in preparation; (Costa e Silva et al. 2009; Fernández et al. 2010; Goulao et al. 2011; Kirst et al. 2004; Mora and Serra 2014; Ruedell et al. 2013) is related to a distinct carbon metabolism in leaves and stems, which differs from the source-to-sink relationship observed in $E$. grandis at the same condition.

\section{Material and methods}

\subsection{Plant material and low temperature treatment}

Seeds of E. globulus and E. grandis, obtained from clonal gardens (respectively GloPirAPSF1 and GraPirAPSF2, Caiçara Sementes-http://www. sementescaicara.com, Araújo et al. 2018) were germinated and cultivated in the greenhouse for 34 weeks. Eight weeks before the beginning of the treatment, plants were transferred to a growth chamber with 12:12 light/dark photoperiod, $500 \mu \mathrm{mol}$ photons $\mathrm{m}^{-2} \mathrm{~s}^{-1}$ and average temperature of $25^{\circ} \mathrm{C}$. After this acclimation period, plants with similar visual height and stem diameter were randomly divided into two groups: (a) control, kept in the same conditions of the acclimation period; and (b) cold treatment, where plants were kept under the same photoperiod and light condition, but the chamber temperature was set to $10{ }^{\circ} \mathrm{C}$, starting with the light period. Leaf and stem samples were harvested at regular intervals: (I) $0.5 \mathrm{~h}$; (II) $6 \mathrm{~h}$; (III) $12 \mathrm{~h}$; (IV) $18 \mathrm{~h}$ and (V) $24 \mathrm{~h}$ after the change in temperature and immediately frozen in liquid nitrogen. Throughout the text we referred to the light period as day and to the dark period as night. Stem samples, counted from the apex to the leaf at height of the fifth node, were collected, as well as all the leaves of this portion. Frozen plant material was stored at $-80{ }^{\circ} \mathrm{C}$ until further analysis. For all analyses, five biological replicates were used, each representing material pooled from five independent plants.

\subsection{Sequence analysis in Forest database and primer design}

The databases Forest, Eucalyptus Genome Network (EUCAGEN, http://www.eucagen.org) (Mizrachi et al. 2010) and Phytozome (http://www.phytozome. net/) were used to search for homologues of AtKIN10 
genes in eucalyptus. The search for sequences was done using conserved regions of the same genes, obtained through the analysis of sequences of Populus and Arabidopsis, found in NCBI database (http:// www.ncbi.nlm.nih.gov/). The amino acid sequences for KIN10 homologous of Arabidopsis thaliana (AEE73609.1; AEE77544.1), Mesembryanthemum crystallinum (AFN89137.1), Malus hupehensis (ABV49061.1), Nicotiana benthamiana (AAX12442.1), Pisum sativum (CAI96819.1), Populus trichocarpa (XP_002306053.1; XP_002319341.1), Solanum berthaultii (AGF39571.1), Solanum lycopersicum (NP_001233965.1), Vicia faba (CAI96818.1) and Zea mays (ACG33875.1; NP_001105673.1) were aligned with the eucalyptus sequences identified here, using the ClustalW included in MEGA 5 software to perform this alignment (Tamura et al. 2011). Next, this alignment was used to construct a phylogenetic tree according to the neighbor-joining algorithm (Saitou and Nei 1987). Bootstrap values were obtained after 5000 replications. Additionally, the online tools Blastx (http://blast.ncbi.nlm.nih.gov/Blast.cgi) and Conserved Domains Database-CDD (http://www.ncbi. nlm.nih.gov/cdd/) were also used to search for KIN10 domains of Arabidopsis thaliana in sequences obtained from Eucalyptus.

The primers for eucalyptus KIN10s genes were designed using CAPE algorithm (BioEdit software) (Hall 1999) and CLUSTALX software (Thompson et al. 1994). Table S1 shows the primer sequences of the genes analyzed in the present study.

\subsection{Gene expression analysis}

The cetyl trimethylammonium bromide (CTAB) method (Quang et al. 2012) was used to extract total RNA, which was further treated with rDNase I (RNase-Free, Bioagency) and quantified in a Qubit fluorometer (Invitrogen). The quality of RNA was inspected in $1 \%$ agarose gel electrophoresis with ethidium bromide and visualization under UV light. cDNA was obtained using SuperScript ${ }^{\mathrm{R}}$ VILO $^{\text {TM }}$ cDNA synthesis (Invitrogen).

Analyzes by qRT-PCR were made in an iQ5 Multicolor Real-Time PCR Detection System (BioRad) using SYBR green PCR Master Mix (Applied Biosystems). Each sample was analyzed with three technical replicates in a reaction mix containing $20 \mu \mathrm{L}$ of reverse-transcribed cDNA from $5 \mathrm{ng}$ total RNA. Isocitrate dehydrogenase (IDH) gene was used as constitutive gene as recommended by Moura et al. (2012) (Table S1) and the relative expression was presented using the method $2^{-\Delta \Delta \mathrm{Ct}}$ (Figs. 1, 2) and $2^{-\Delta \mathrm{Ct}}$ (Figures S4 and S5), according to Livak and Schmittgen (2001).

\subsection{Extraction and identification of major polar metabolites}

Primary metabolites extraction and derivatization were carried out in the same samples used for molecular analysis, following the protocol described by Lisec et al. (2006). Briefly, $50 \mathrm{mg}$ of frozen plant material (leaves and stems) were used for each biological sample. Extraction in $700 \mu \mathrm{l}$ of methanol per sample, with shaking at $70{ }^{\circ} \mathrm{C}$, was performed for $1 \mathrm{~h}$. Ribitol was used as internal standard. After centrifugation at $15,300 \times g$, the supernatant was taken, following by the separation of polar and apolar phases by adding chloroform $(300 \mu \mathrm{l})$ and water $(300 \mu \mathrm{L})$ to the supernatant. After a new centrifugation, $1 \mathrm{~mL}$ of the polar phase was taken and reduced by dryness. Derivatization and analysis by gas chromatography coupled to mass spectrometry (GC-MS) were performed according to the same work (Lisec et al. 2006). Data were normalized and transformed as described by Lisec et al. (2006) and Caldana et al. (2013). Total protein (Bradford 1976) and starch content (Critchley et al. 2001) were analyzed according to standard protocols described in the literature.

\subsection{Statistical analyses}

Results were expressed as mean \pm standard error and were compared by the Tukey test at $5 \%$ probability. The relationship between gene expression and identified metabolites were evaluated through Pearson correlation test at 5\% probability (Ruprecht and Persson 2012). For both analyzes, we used GraphPad Prism software, version 8.00 for Windows (Microsoft), GraphPad Software, San Diego, California, USA. Principal components analysis (PCA) was carried out using $R$ software version 2.12.2. (http:// www.r-project.org), with criteria established by Ruprecht et al. (2011). 

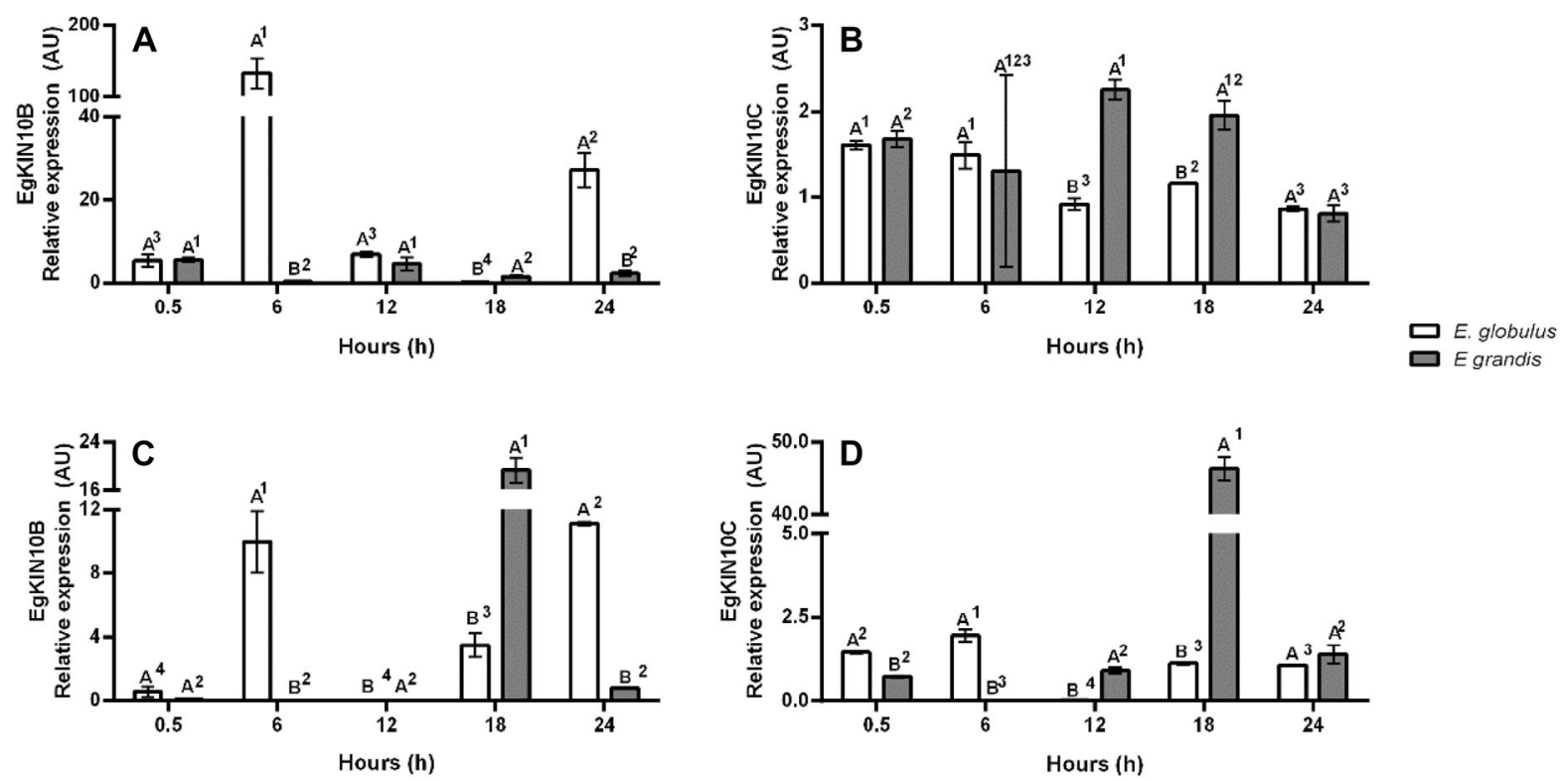

Fig. 1 Relative expression of the genes (a), (c) KIN1OB and (b), (d) KIN10C in (a), (b) leaves and (c), (d) stems of $E$. globulus and E. grandis harvested over $24 \mathrm{~h}$ of exposure at $10{ }^{\circ} \mathrm{C}$. Letters indicate statistical differences between species within the same harvesting time and numerals indicate

differences between harvesting times within the same species and treatment. Experiment performed with triplicates. Differences among experimental groups evaluated by One-way ANOVA with Tukey test at 5\% probability. Error bars: standard error
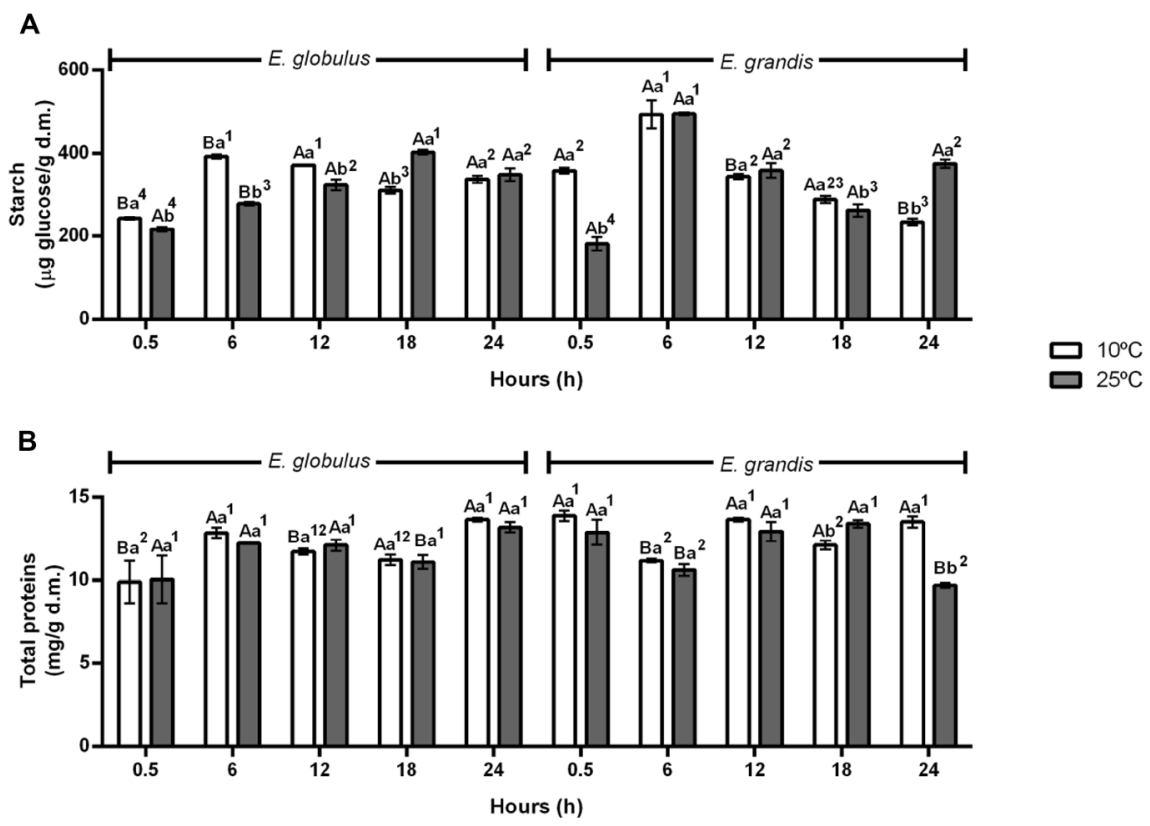

Fig. 2 Content of (a) starch and (b) total proteins in leaves of $E$. globulus and E. grandis maintained at $10{ }^{\circ} \mathrm{C}$ and $25^{\circ} \mathrm{C}$ over $24 \mathrm{~h}$. Capital letters indicate statistical differences between species within the same harvesting time and treatment; lower case letters indicate differences between treatments for the same harvesting timepoints and species; numerals indicate differences between harvesting timepoints within the same species and treatment. Experiment performed with five biological samples. Differences among experimental groups evaluated by One-way ANOVA with Tukey test at $5 \%$ probability Error bars: standard error 


\section{Results}

\subsection{Identification of Eucalyptus genes homologous to AtKIN10}

Two complete cDNA sequences of Eucalyptus grandis genes homologous to AtKIN1O (EgKINIOB and EgKIN10C) were recovered from search of public databases. Using the online tools Blastx (http://blast. ncbi.nlm.nih.gov/Blast.cgi) and Conserved Domains Database-CDD (http://www.ncbi.nlm.nih.gov/cdd/) it was possible to identify Arabidopsis thaliana domains in sequences obtained from Eucalyptus (Figures S1 and S2), suggesting their homology to AtKIN10. Additionally, comparisons of the deduced protein sequence with sequences already characterized in A. thaliana, Mesembryanthemum crystallinum, Malus hupehensis, Nicotiana benthamiana, Pisum sativum, Populus trichocarpa, Solanum berthaultii, Solanum lycopersicum and Zea mays emphasized this fact (Figures S2 and S3).

\subsection{Expression of $K I N$ genes}

KIN1OB showed higher expression in leaves of $E$. globulus than in E. grandis in response to cold (Fig. 1a and Fig. S4A). Moreover, leaves of E. globulus showed a considerable daily variation in KIN1OB expression with a maximum soon after the onset of exposure to cold, a subsequent fall throughout the day and a new increase just before dawn of the next day. KIN10C (Fig. 1b and Fig. S4B) had a more modest relative expression and few changes throughout the day in leaves of E. globulus when exposed to cold; the same occurs in E. grandis during the light period, with an increased expression during the first $6 \mathrm{~h}$ of dark and subsequent decline until the end of cold treatment.

The expression profiles of KIN1OB and KIN10C genes were similar in stems of $E$. grandis plants (Fig. 1c, d and Figs. S5A and S5B, respectively); a maximum relative expression was observed after $18 \mathrm{~h}$ of exposure to cold and few differences were observed at other harvest times. Both genes also showed a similar pattern in E. globulus stems exposed to cold with: higher values during the daytime period, and a reduction of the relative expression in transition to night, with a second increase at the end of treatment.

\subsection{Primary metabolism}

\subsubsection{Macromolecules quantification: starch and proteins}

In general, irrespective of the thermal treatment, $E$. globulus had less starch in leaves when compared to $E$. grandis during the day, with the inverse behavior observed during the night (Fig. 2a). Moreover, E. globulus leaves exhibited lower oscillation in starch content over 24 h compared to E. grandis. By contrast, leaves of E. grandis exposed to $10{ }^{\circ} \mathrm{C}$ showed a marked decrease in the starch content, with the lowest level observed after $24 \mathrm{~h}$ of treatment.

While the amount of foliar protein in E. globulus did not dramatically change over $24 \mathrm{~h}$ in the control $\left(25^{\circ} \mathrm{C}\right)$ and treated $\left(10^{\circ} \mathrm{C}\right)$ plants (Fig. 2b), leaves of E. grandis generally exhibited higher leaf protein content than E. globulus at both temperatures.

In stems, starch (Fig. 3a) and total protein (Fig. 3b) contents were higher in E. grandis samples when compared to E. globulus, regardless of harvest timepoint or thermal treatment. E. grandis had the highest accumulation of starch $6 \mathrm{~h}$ after beginning of cold treatment, and later falling during the night period. $E$. globulus also showed maximum accumulation at $6 \mathrm{~h}$ of cold exposure.

Total protein content of both species oscillated less in stem samples when compared to leaves. For $E$. globulus, the main difference between thermal treatments was the moment when protein levels were at their lowest: plants exposed to $10{ }^{\circ} \mathrm{C}$ showed a reduction of total protein content in transition from day to night ( $12 \mathrm{~h}$ after the start of treatment), while the same occurred $6 \mathrm{~h}$ earlier in plants exposed to $25{ }^{\circ} \mathrm{C}$.

\subsubsection{Primary metabolome analyses}

Using these same samples, characterization of several primary metabolites in polar extracts was carried out by GC-MS, identifying 44 compounds (Figs. 4, 5, Figures S4 to S7 and Dataset S1): 12 amino acids, 12 carbohydrates, eight organic acids, six phenolic compounds, four polyols and two miscellaneous compounds (putrescine and phosphoric acid).

In general, $10{ }^{\circ} \mathrm{C}$ exposure mostly affected leaf amino acids and carbohydrates, with a large reduction in isoleucine, leucine and valine levels occurring in 
A

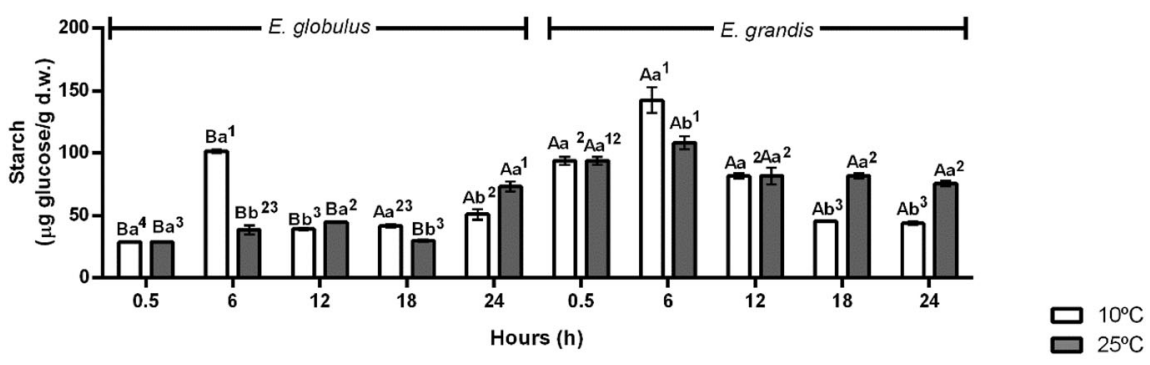

B

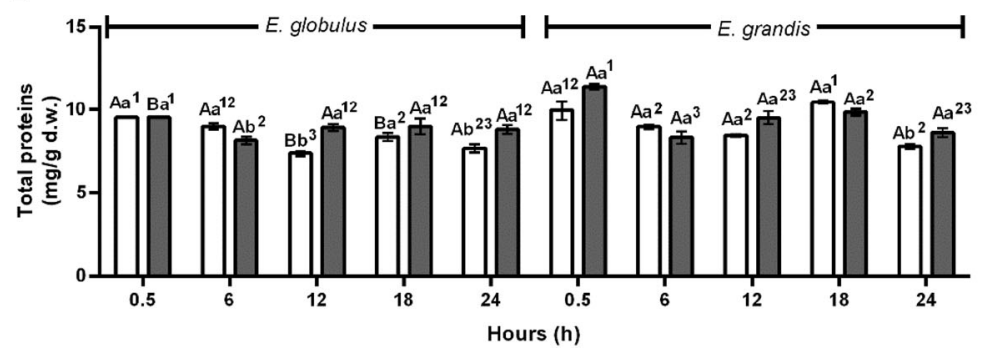

Fig. 3 (a) Starch and (b) total proteins contents in stems of $E$. globulus and E. grandis maintained at $10{ }^{\circ} \mathrm{C}$ and $25^{\circ} \mathrm{C}$ over $24 \mathrm{~h}$. Capital letters indicate statistical differences between species within the same harvesting time and treatment; lower case letters indicate differences between treatments for the same harvesting timepoints and species; numerals indicate

leaves of E. globulus at the end of the 24 h of exposure to cold (Fig. 4). While leaf sucrose levels were similar irrespective of species, harvest timepoints or thermal treatment (Fig. 6b), glucose content differed between the two species. E. globulus displayed increase of glucose levels shortly after $6 \mathrm{~h}$ of treatment, with subsequent stabilization in plants exposed to $10{ }^{\circ} \mathrm{C}$ (Fig. 6a) and subsequent decrease in plants exposed at $25^{\circ} \mathrm{C}$. E. grandis displayed the reverse response, while plants kept at $10{ }^{\circ} \mathrm{C}$ showed an increase in glucose content only at the end of the night, the same compound accumulated at higher levels at $25^{\circ} \mathrm{C}$ during the first harvest timepoint, with a subsequent fall and stabilization (Figs. 4, 5, 6).

Less intense changes throughout the day were observed for organic acid profiles in leaves (Dataset S1). However, a reduction of compounds of the tricarboxylic acid (TCA) cycle, i.e., citrate, malate and, in a lesser extent, succinate, is notable in $E$. globulus samples harvested in the late evening (Fig. 4 and S4). When both species were maintained at $10{ }^{\circ} \mathrm{C}$, larger amounts of citrate and malate were observed at the end of the night period. differences between harvesting timepoints within the same species and treatment. Experiment performed with five biological samples. Differences among experimental groups evaluated by One-way ANOVA with Tukey test at $5 \%$ probability Error bars: standard error

In stems, many amino acids and carbohydrates were present in larger amounts early in the morning with a reduction in content occurring throughout the day, with this pattern being more intense for amino acids of E. globulus and for carbohydrates of E. grandis when both species were kept at $25^{\circ} \mathrm{C}$ (Fig. 5, S6 and S7 and Dataset S1). Additionally, less severe oscillations than those presented in leaves also occurred among organic acids, phenolic compounds, polyols and other compounds identified in stems samples, regardless of species and temperature treatment (Figures S6 and S7).

The levels of the major sugars present in stems of both species (Fig. 7) oscillated more intensely than in leaves, responding to the variations of the day and the environmental temperature to which plants were exposed. Glucose content (Fig. 7a) in E. globulus stems was reduced during night for both temperature treatments, with more pronounced reduction in plants exposed to $25{ }^{\circ} \mathrm{C}$. Throughout the day, accumulation of this hexose was less intense in stems exposed to $10^{\circ} \mathrm{C}$. Samples of E. grandis displayed opposite pattern of change, with plants kept at $10{ }^{\circ} \mathrm{C}$ showing 


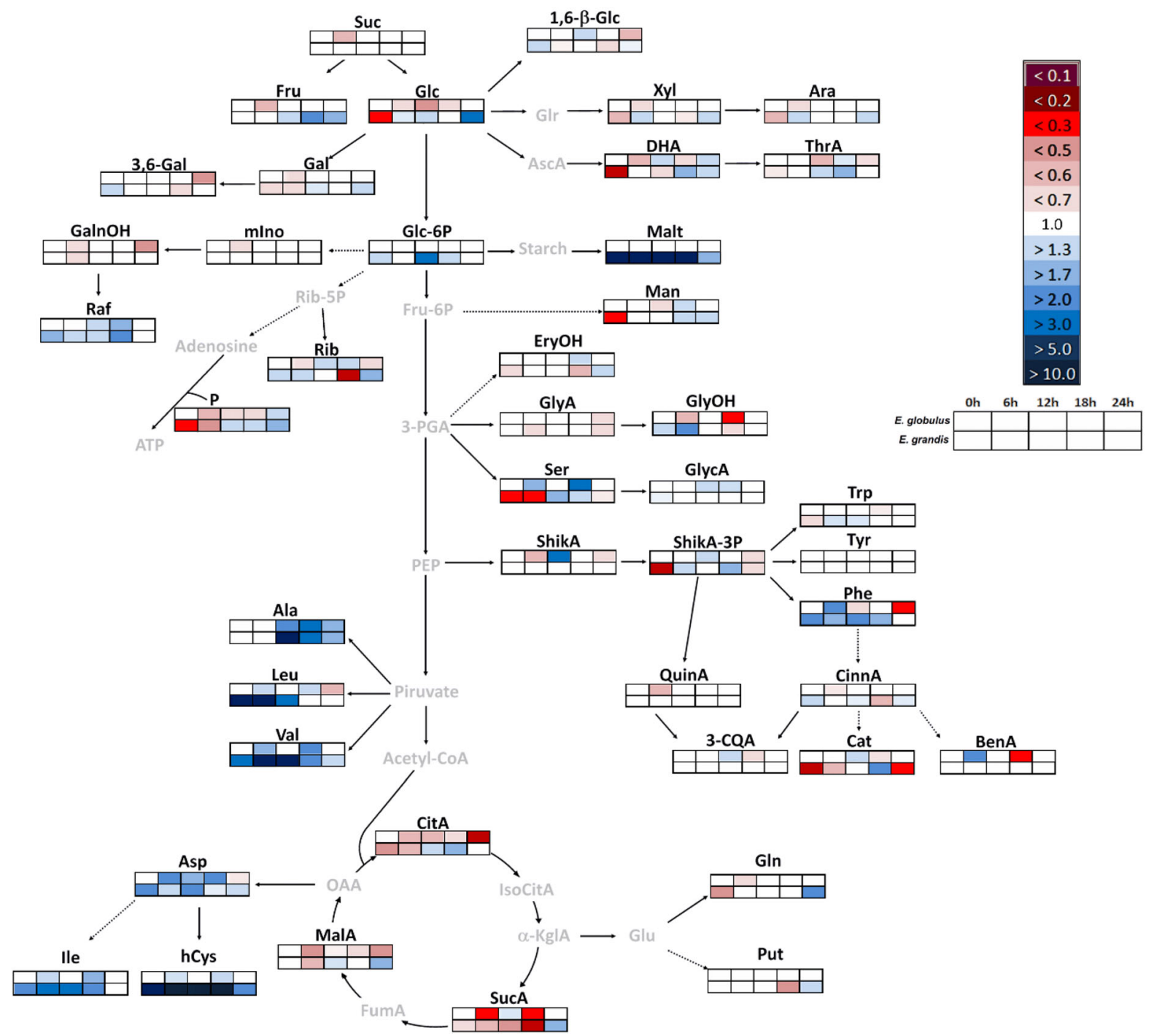

Fig. 4 Main metabolites identified in leaf samples of $E$. globulus (upper squares) and E. grandis (lower squares), according to the harvest time. Values relatives to control treatment $\left(25^{\circ} \mathrm{C}\right)$. Shades of blue indicate higher relative values observed in samples exposed to cold nights; red shades indicate

small oscillations in their glucose content during day and an increase at night.

Sucrose levels displayed small oscillations throughout the day for samples of E. globulus exposed at $10{ }^{\circ} \mathrm{C}$ (Fig. 7b) and were essentially invariant in $E$. grandis samples under the same conditions. Small oscillations were also observed in both species exposed to $25^{\circ} \mathrm{C}$, but, unlike at $10{ }^{\circ} \mathrm{C}$, the amount of sucrose tended to be higher in E. globulus compared to $E$. grandis during the daytime period. lower relative values observed in samples exposed to cold nights. Compounds in gray color were not identified. Abbreviations of the compounds are identified in Table S1. (Color figure online)

\subsection{Multivariate analyses}

Significant negative relationships $(-0.6<\mathrm{R}<-0.3)$ between starch and sucrose levels were identified in leaves of $E$. globulus exposed to $10{ }^{\circ} \mathrm{C}$, while its relations with glucose and fructose was strongly positive $(+0.6<\mathrm{R}<+1.0)$ at the same temperature condition for this specie at $10{ }^{\circ} \mathrm{C}$. For $E$. grandis, starch only correlated positively (+ 


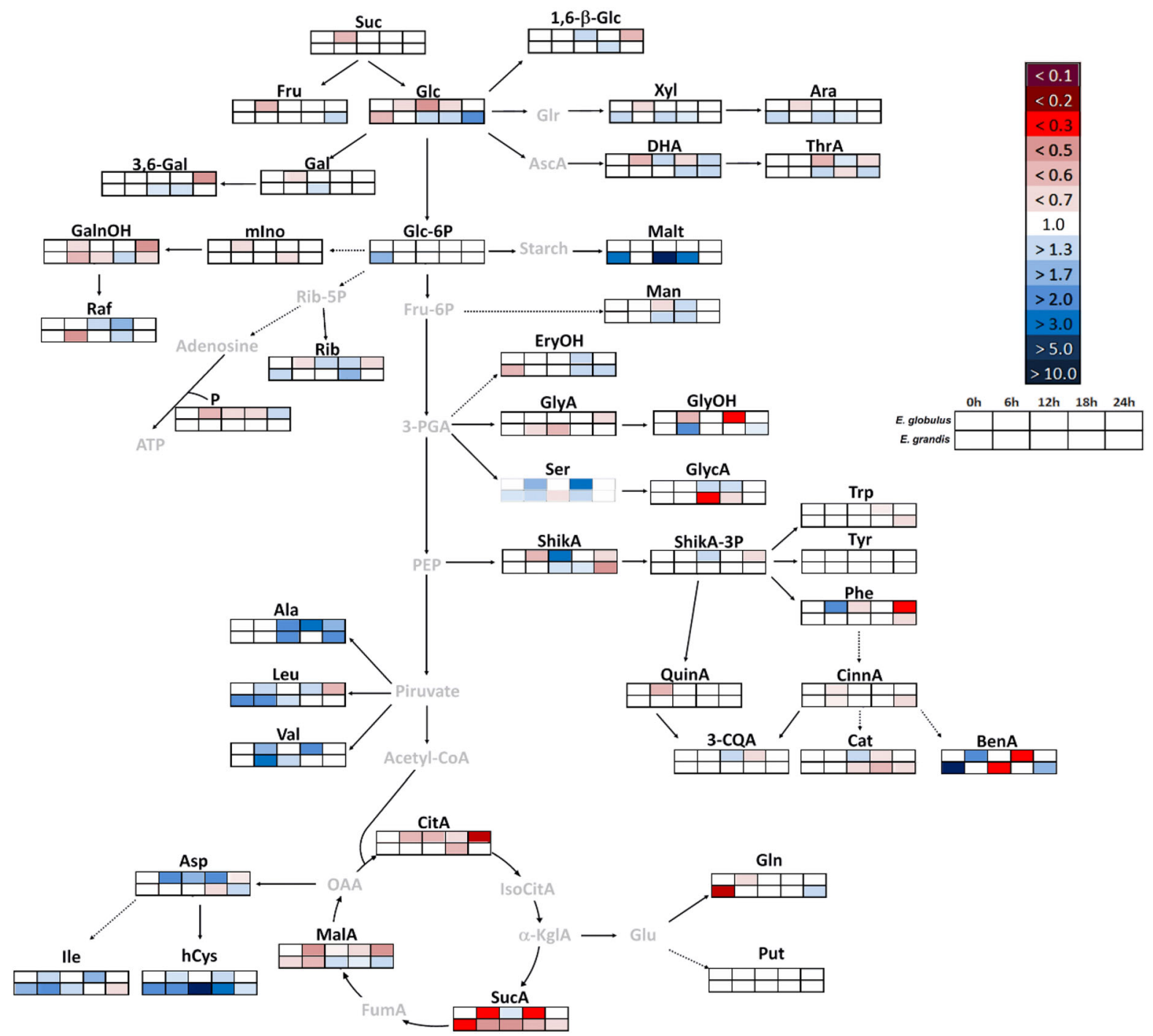

Fig. 5 Main metabolites identified in stem samples of $E$. globulus (upper squares) and E. grandis (lower squares), according to the harvest time. Values relatives to control treatment $\left(25^{\circ} \mathrm{C}\right)$. Shades of blue indicate higher relative values observed in samples exposed to cold nights; red shades indicate

$0.6<\mathrm{R}<+1.0$ ) with $K I N$ gene expression at the same thermal treatment.

In stems of E. globulus plants exposed to $10{ }^{\circ} \mathrm{C}$, the total protein content positively correlated (+ $0.6<\mathrm{R}<+1.0$ ) with the amount of different amino acids (i.e., BCAAs, asparagine, phenylalanine and tryptophan), as well as some carbohydrates (i.e., glucose, maltose and mannose). In relation to starch content, there were practically no significant lower relative values observed in samples exposed to cold nights. Compounds in gray color were not identified. Abbreviations of the compounds are identified in Table S1. (Color figure online)

relationships with any other metabolic parameters for E. globulus in both treatments.

A principal component analysis (PCA) was performed with metabolome dataset obtained (leaves represented in Figures S8 and stems in Figure S9). A clear distinction of each experimental group was observed for any combination of the three main principal components (PCs). For leaves, the differences between the thermal treatments predominate (i.e., factor best explained by PC1, Figure S8), while 
A

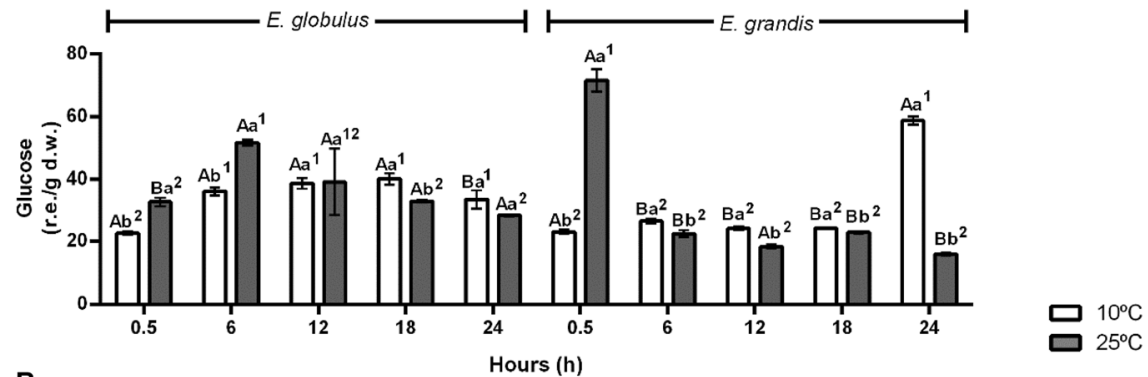

B

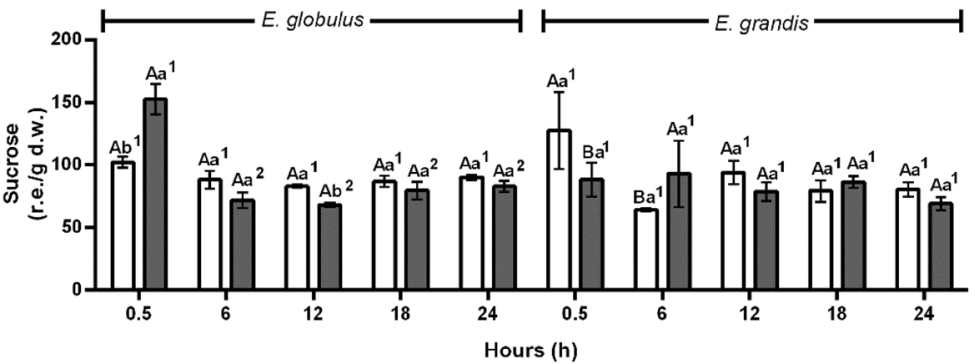

Fig. 6 Relative content of (a) glucose and (b) sucrose in leaves of E. globulus and E. grandis maintained at $10^{\circ} \mathrm{C}$ and $25^{\circ} \mathrm{C}$ over $24 \mathrm{~h}$. Capital letters indicate statistical differences between species within the same harvesting time and treatment; lower case letters indicate differences between treatments for the same harvesting timepoints and species; numerals indicate differences between harvesting timepoints within the same species and treatment. Experiment performed with five biological samples. Differences among experimental groups evaluated by One-way ANOVA with Tukey test at 5\% probability Error bars: standard error
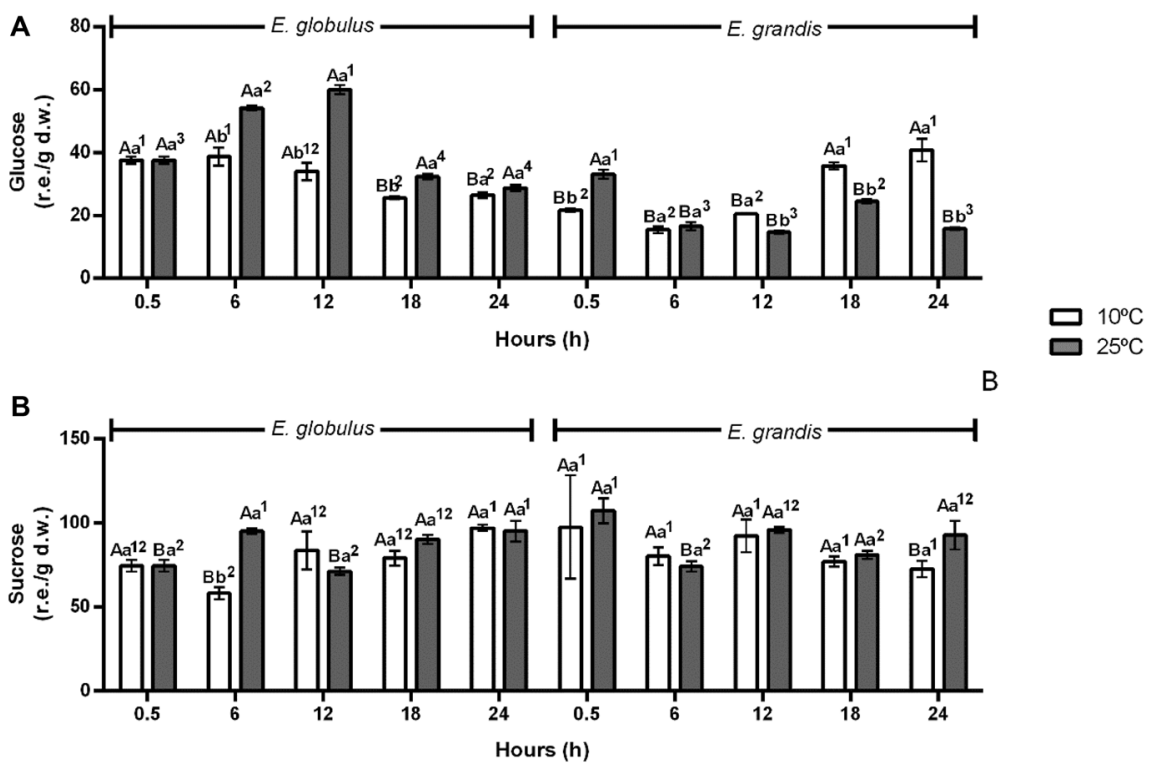

Fig. 7 Relative content of (a) glucose and (b) sucrose in stems of E. globulus and E. grandis maintained at $10^{\circ} \mathrm{C}$ and $25^{\circ} \mathrm{C}$ over $24 \mathrm{~h}$. Capital letters indicate statistical differences between species within the same harvesting time and treatment; lower case letters indicate differences between treatments for the same harvesting timepoints and species; numerals indicate differences between harvesting timepoints within the same species and treatment. Experiment performed with five biological samples. Differences among experimental groups evaluated by One-way ANOVA with Tukey test at $5 \%$ probability Error bars: standard error 
for stems (Figure S9), interspecific differences provide the best separation in PC1. Moreover, it should be noted that, for stem samples thermal, differences were well explained by the components present in PC2.

\section{Discussion}

\subsection{Relationship between sugar profile and expression of KIN genes}

Although the main carbohydrates profile in leaves and stems (Figs. 2a, 3a, 6, 7) of Eucalyptus respond similarly to cold exposure, the manner how they do so is tissue-dependent. Such behavior was already expected, given the functional differences of each organ (Bauerfeind et al. 2015). Furthermore, this behavior is consistent with expression pattern of KIN10 genes (Fig. 1), a major component of catalytic complex of SnRK1 system (Tomé et al. 2014; Williams et al. 2014).

In that way, it is important to highlight that in situations of low energy availability, SnRK1 is responsible for activating and regulating genes related to numerous catabolic pathways, accessing alternative sources of energy and substrates and suppressing several pathways with a high energy demand (Tomé et al. 2014; Wurzinger et al. 2018). Nonetheless, the activity of the SnRK1 system is a highly complex process, controlling, and in turn being controlled by, a myriad of regulatory proteins and signaling metabolites such as soluble sugars and also being dependent on the tissue nature investigated (Halford and Hey 2009; Lunn et al. 2014; Smeekens et al. 2010).

Therefore, the greater expression of leaf KIN genes during the day (Fig. 1a, b) may be especially important in E. globulus which displayed no significant increase in amount of the major carbohydrates (i.e., glucose and sucrose) in their leaves during the daytime under cold conditions, even though this species displays a higher rate of carbon assimilation when exposed to low temperatures (Domingues-Junior et al., MS in preparation). One possible explanation for this phenomena would be a greater sink strength of the stem, stimulated by cold signaling and starting to consume high quantities of photoassimilates (Arias et al. 2014; Campoe et al. 2013; Çetinkol et al. 2012). In this sense, it is worth mentioning that E. globulus shows a faster growth rate compared to E. grandis when both species are exposed to low temperatures (Costa e Silva et al. 2009; Fernández et al. 2010; Goulao et al. 2011; Kirst et al. 2004; Mora and Serra 2014; Moura et al. 2010; Ruedell et al. 2013). Moreover, this hypothesis is consistent with observations described in Fig. 1c, d, wherein stems of this species also displayed greater expression of KIN1O genes during day, suggesting a higher demand for energy resources.

In E. grandis, leaves and stems kept at $10{ }^{\circ} \mathrm{C}$ showed higher expression of KIN10 during the night. Associating this with the stressful impact that cold exerts on the development of this species (Budzinski et al. 2016) and the small oscillation observed in carbohydrate levels at night (Figs. 6 and 7), we postulate that the greater expression of KIN10 genes favors the increase of available carbon skeletons which might be used as an energy supply and/or as well as substrate to the biosynthesis of defense metabolites.

We, therefore, suggest that the two species differ in the way they coordinate the activation of the SnRK1 system with sugar metabolism and vegetative growth when exposed to low temperatures. For E. globulus, this system is directed to a higher carbon assimilation and greater stem growth rate during the day, while in E. grandis, it would appear to be associated to providing substrates for responses against cold (Hoermiller Imke et al. 2017; Tomé et al. 2014). However, future studies seeking the association of this pattern with the response of genes related to the cell cycle, the activity of metabolic protection systems (such as antioxidant enzymes), as well as a prolonged low temperature exposure will allow the confirmation or refutation of the hypothesis raised here.

\subsection{Cold provokes different changes in primary metabolism of E. globulus and E. grandis}

The development and widespread adoption of gas chromatography mass spectrometry (GC-MS) has facilitated numerous studies characterizing the metabolic profiles of different plant species in response to endogenous and external alterations (Bauerfeind et al. 2015; Dhuli et al. 2014; Mokochinski et al. 2018; Tarkowski and Van den Ende 2015). However, the majority of the studies to date have focused on herbaceous plants, with only a few being related to tree species (Correia et al. 2018; Dhuli et al. 2014; Warren 
A

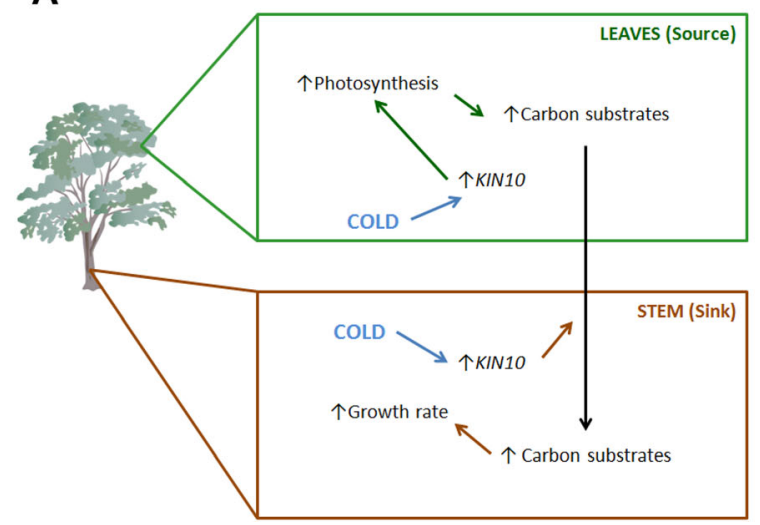

Fig. 8 Relations between source and sink tissues of (a) $E$. globulus and (b) E. grandis when both species are exposed to $10{ }^{\circ} \mathrm{C}$. This schematic representation combines molecular and metabolic data of the present work and observations discussed in other work of our group (Domingues-Junior et al., MS in preparation). Small arrows indicate changes in parameters

et al. 2012; Warren 2011). To our knowledge, the present study is the first to analyze daily profile of the main central metabolites in leaves and stems of Eucalyptus exposed to low temperatures.

The compounds presented herein (Figs. 4, 5 and Dataset S1) comprise several metabolic classes, including non-structural carbohydrates, amino acids, organic acids, polyols and two compounds classified as "miscellaneous" (putrescine and phosphate). In general, the division between periods of light and dark significantly impacted levels of the different metabolites described in this study.

When analyzing each metabolite in the context of the biochemical class to which it belongs (Figures S4 to S7), a similar pattern was observed between $E$. globulus and E. grandis. The metabolite oscillations were smaller in stems when compared to leaves and plants exposed to $10{ }^{\circ} \mathrm{C}$ displayed higher levels of free amino acids, especially of the branched-chain amino acids (BCAAs, i.e. leucine, isoleucine and valine) (Figs. 4, 5). According to Avin-Wittenberg et al. (2012) and Tomé et al. (2014), stress conditions are responsible for increasing the content of free amino acids, as a result of either enhanced degradation and/or reduced synthesis of proteins. Moreover, free BCAAs can be catabolized in order to maintain the levels of the TCA cycle intermediates and to provide electrons to mitochondrial electron carrier chain (Arias et al. 2014; Avin-Wittenberg et al. 2012; Budzinski et al. 2016). In this sense, the increase of citrate, malate and succinate

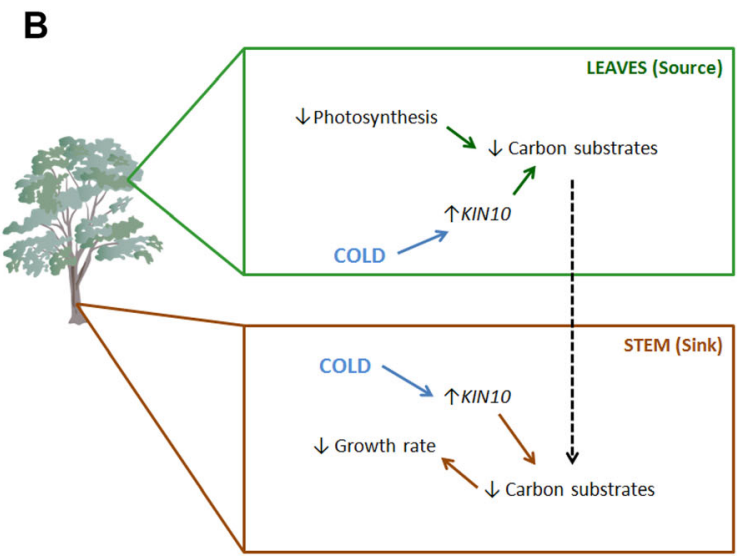

values in plants exposed to cold $\left(10^{\circ} \mathrm{C}\right)$ compared to plants of control group $\left(25^{\circ} \mathrm{C}\right)$ : (upward arrow) indicates increased values of those parameters and (downward arrow) indicates decreased values of those parameters. Dashed line indicates reduction of carbon substrates available to exportation from source to sink tissues

at the end of the night period is consistent with the decline observed in BCAA content in the leaves of both eucalyptus species following exposure to cold.

Similar changes in profile of BCAAs and TCA cycle components were described in plants of pea submitted to conditions of low energy availability, being regulated by the SnRK1 system (Radchuk et al. 2010). Thus, changes in levels of amino and organic acids observed in leaves and stems of E. globulus and E. grandis exposed to $10{ }^{\circ} \mathrm{C}$ would be anticipated, given the changes observed here in KIN10 gene expression (already discussed above). However, no other relationships between the content of these metabolites and the expression of the KIN1O genes were observed. We, therefore, postulate the existence of additional factors, not analyzed in the present work, including the possibility of increased activity of degradative enzymes activity or post-translational regulatory events of this process, as links between the initial signaling via SnRK1 and the catabolic response. Future studies should encompass analysis at multiple different levels namely transcriptomic, proteomic and metabolomic levels in order to gain a more detailed insight into the underlying mechanisms.

The principal component analysis (PCA) of the metabolic profile data revealed a clear distinction between species and thermal environments (Figures S8 and S9), indicating that, even for a short period, cold exposure was able to evoke distinct responses in $E$. globulus and E. grandis plants. When analyzing 
variables according to the tissues, we can observe a greater susceptibility of photoassimilates sources organs (leaves) to thermal treatments. In this manner, TCA cycle components, BCAAs and glucose were the main components responsible for this response (Figure S8 and Table S3). Such behavior is perfectly plausible given the high sensitivity of both carbon assimilatory and respiratory processes in leaves exposed to low temperatures (Navarrete-Campos et al. 2013; Tarkowski and Van den Ende 2015). Also, leaf, working as the main organ for perception of external environment and converging thermal, nutritional and luminous signals must respond immediately to any change in its environment, in order to generate both rapid and long term responses (Tomé et al. 2014).

Moreover, the lack of separation of temperature treatments according to PC1 for stem metabolites (Figure S9 and Table S4) and the lower number of cold induced changes observed on those variables suggest a lower susceptibility of stems to the thermal environment (at least, to the conditions presented in this work). Moreover, the responses observed for stem samples indicate that intrinsic differences found in $E$. globulus and E. grandis are larger and more significant than changes evocated by the thermal environment imposed here. It is probably due the fact that stem is mainly composed of vascular tissues, where cell walls are important sink of carbon (Allahverdiyeva et al. 2015; Epron et al. 2012); therefore, the biosynthesis of their component affects entire development, once it exerts great influence on the plant energy balance, and must be under strict metabolic and genetic control to allow the best performance according to the environmental conditions.

Therefore, we could observe in this work that the expression of KIN1O genes in leaves and stems of $E$. grandis is consistent with their basal function (being active in situations of energy deficit) (Tomé et al. 2014; Williams et al. 2014; Wurzinger et al. 2018); however, in E. globulus, the expression of those genes during the day and under cold, a condition considered not harmful for its vegetative growth, suggests two possible scenarios: (I) there is little energy available from photosynthesis; or (II) the converted energy is rapidly consumed. Moreover, the soluble sugar contents suggest that the increase of glucose in E. grandis stems at cold night period is consistent with the higher expression of KIN10 genes soon after the transition to the dark period, meaning that, with KIN10 genes signaling, catabolic pathways would guarantee glucose supply for the nocturnal period (Tomé et al. 2014; Williams et al. 2014; Wurzinger et al. 2018) in stems of this species. By other way, in E. globulus plants exposed to cold, the small increase of leaf glucose, associated with the absence of oscillation in sucrose levels and starch content, suggests the existence of a strong photoassimilate sink (Wu and Birch 2010). Moreover, our group has observed a high photosynthetic rate when E. globulus is maintained at $10{ }^{\circ} \mathrm{C}$ (Domingues-Junior et al., MS in preparation), thus higher rate of photoassimilate synthesis is expected under this condition. In this manner, the small decrease in sucrose content summed to the absence of oscillation of glucose levels in E. globulus stems suggests an increased sink strength (Wu and Birch 2010), possibly by investing in a higher cell wall deposition and growth (Cerasuolo et al. 2016; Osakabe et al. 2013), behavior possibly triggered by low temperature signaling.

In that way, we could observe that cold $\left(10{ }^{\circ} \mathrm{C}\right)$ was able to promote a higher KIN10 gene expression in leaves of E. globulus, stimulating photosynthesis and promoting photoassimilates production. Another work from our group and carried out parallel to the present study (Domingues-Junior et al., MS in preparation) has observed that the same low temperature conditions applied here were able to promote a higher stem growth rate in E. globulus when this species were exposed to cold for 30 days. Therefore, this higher stem growth rate triggered by low temperature signaling probably increases the sink strength of this tissue, consuming the photoassimilate surplus and thereby maintaining a higher growth rate. If this scenario is maintained over time, such dynamics would guarantee the higher vegetative development of E. globulus when plants of the species are subjected to low temperatures. A schematic representation of this hypothesis is presented in Fig. 8.

In conclusion, the present work is the first to identify changes on primary metabolic profile of source and sink tissues of two Eucalyptus species when exposed to $10{ }^{\circ} \mathrm{C}$ for 1 day cycle. The fact that it was possible to distinguish species according to the metabolic profile and the content of important carbon sinks (starch and total protein content) suggests starting points for future studies, in which such interactions between primary metabolism and cell wall biosynthesis should be carefully analyzed in 
order to select desirable parameters for higher production of cellulose and biofuels from Eucalyptus.

Acknowledgements Open access funding provided by Max Planck Society. We acknowledge Laerti Reis Roque, Luana Reis Roque, Dulcinéia Pereira de Souza, Änne Eckardt, Gudrun Wolter for exceptional technical assistance. This work was supported by funding from the São Paulo Research Foundation (FAPESP, Grant Numbers 2014/01200-6 and 2018/20572-2 to A.P.D.-J.; 2011/51949-5 to P.M.) and the Brazilian Council for Scientific and Technological Development (CNPq Grant Number 246374/2012-8 to L.R.S.).

Author contributions APDJ, PM and ARF designed the experiments; APD-J, DDM, MM, LR-S and LPS carried out the experiments; APD-J, ARF and PM wrote the paper.

\section{Compliance with ethical standards}

Conflict of interest The authors declare no conflicts of interest.

Open Access This article is distributed under the terms of the Creative Commons Attribution 4.0 International License (http:// creativecommons.org/licenses/by/4.0/), which permits unrestricted use, distribution, and reproduction in any medium, provided you give appropriate credit to the original author(s) and the source, provide a link to the Creative Commons license, and indicate if changes were made.

\section{References}

Allahverdiyeva Y, Battchikova N, Brosché M, Fujii H, Kangasjärvi S, Mulo P, Mähönen AP, Nieminen K, Overmyer K, Salojärvi J, Wrzaczek M (2015) Integration of photosynthesis, development and stress as an opportunity for plant biology. New Phytol 208:647-655

Araújo P, Cesarino I, Mayer JLS, Ferrari IF, Kiyota E, Sawaya ACHF, Leme AFP, Mazzafera P (2014) A model system to study the lignification process in Eucalyptus globulus. Physiol Plant 152:17-31

Arias MC, Pelletier S, Hilliou F, Wattebled F, Renou J-P, D'Hulst C (2014) From dusk till dawn: the Arabidopsis thaliana sugar starving responsive network. Front Plant Sci 5:482

Avin-Wittenberg T, Tzin V, Angelovici R, Less H, Galili G (2012) Deciphering energy-associated gene networks operating in the response of Arabidopsis plants to stress and nutritional cues. Plant J 70:954-966

Baena-Gonzalez E, Rolland F, Thevelein JM, Sheen J (2007) A central integrator of transcription networks in plant stress and energy signalling. Nature 448:938-942

Bauerfeind MA, Winkelmann T, Franken P, Druege U (2015) Transcriptome, carbohydrate and phytohormone analysis of Petunia hybrida reveals a complex disturbance of plant functional integrity under mild chilling stress. Front Plant Sci 6:583
Bechtold U, Field B (2018) Molecular mechanisms controlling plant growth during abiotic stress. J Exp Bot 69:2753-2758

Bradford MM (1976) A rapid and sensitive method for the quantitation of microgram quantities of protein utilizing the principle of protein-dye binding. Anal Biochem 72:248-254

Budzinski IGF, Moon DH, Morosini JS, Lindén P, Bragatto J, Moritz T, Labate CA (2016) Integrated analysis of gene expression from carbon metabolism, proteome and metabolome, reveals altered primary metabolism in Eucalyptus grandis bark, in response to seasonal variation. BMC Plant Biol 16:149

Caldana C, Li Y, Leisse A, Zhang Y, Bartholomaeus L, Fernie AR, Willmitzer L, Giavalisco P (2013) Systemic analysis of inducible target of rapamycin mutants reveal a general metabolic switch controlling growth in Arabidopsis thaliana. Plant J 73:897-909

Campoe OC, Stape JL, Nouvellon Y, Laclau J-P, Bauerle WL, Binkley D, Le Maire G (2013) Stem production, light absorption and light use efficiency between dominant and non-dominant trees of Eucalyptus grandis across a productivity gradient in Brazil. For Ecol Manag 288:14-20

Cerasuolo M, Richter GM, Richard B, Cunniff J, Girbau S, Shield I, Purdy S, Karp A (2016) Development of a sinksource interaction model for the growth of short-rotation coppice willow and in silico exploration of genotype $\times$ environment effects. J Exp Bot 67:961-977

Çetinkol ÖP, Smith-Moritz AM, Cheng G, Lao J, George A, Hong K, Henry R, Simmons BA, Heazlewood JL, Holmes BM (2012) Structural and chemical characterization of hardwood from tree species with applications as bioenergy feedstocks. PLoS ONE 7:e52820

Correia B, Hancock RD, Amaral J, Gomez-Cadenas A, Valledor L, Pinto G (2018) Combined drought and heat activates protective responses in eucalyptus globulus that are not activated when subjected to drought or heat stress alone. Front Plant Sci 9:819

Costa e Silva F, Shvaleva A, Broetto F, Ortuño MF, Rodrigues ML, Almeida MH, Chaves MM, Pereira JS (2009) Acclimation to short-term low temperatures in two Eucalyptus globulus clones with contrasting drought resistance. Tree Physiol 29:77-86

Critchley JH, Zeeman SC, Takaha T, Smith AM, Smith SM (2001) A critical role for disproportionating enzyme in starch breakdown is revealed by a knock-out mutation in Arabidopsis. Plant J 26:89-100

Dhuli P, Rohloff J, Strimbeck GR (2014) Metabolite changes in conifer buds and needles during forced bud break in Norway spruce (Picea abies) and European silver fir (Abies alba). Front Plant Sci 5:706

Epron D, Nouvellon Y, Ryan MG (2012) Introduction to the invited issue on carbon allocation of trees and forests. Tree Physiol 32:639-643

Fenollosa E, Gámez A, Munné-Bosch S (2018) Plasticity in the hormonal response to cold stress in the invasive plant Carpobrotus edulis. J Plant Physiol 231

Fernández M, Villarroel C, Balbontín C, Valenzuela S (2010) Validation of reference genes for real-time qRT-PCR normalization during cold acclimation in Eucalyptus globulus. Trees 24:1109-1116 
Goulao LF, Vieira-Silva S, Jackson PA (2011) Association of hemicellulose- and pectin-modifying gene expression with Eucalyptus globulus secondary growth. Plant Physiol Biochem 49:873-881

Halford Nigel G, Hey Sandra J (2009) Snf1-related protein kinases (SnRKs) act within an intricate network that links metabolic and stress signalling in plants. Biochem $\mathrm{J}$ 419:247-259

Hall TA (1999) BioEdit: a user-friendly biological sequence alignment editor and analysis program for Windows 95/98/ NT. Nucleic Acids Symp Ser 41:95-98

Hao X, Wang B, Wang L, Zeng J, Yang Y, Wang X (2018) Comprehensive transcriptome analysis reveals common and specific genes and pathways involved in cold acclimation and cold stress in tea plant leaves. Sci Hortic 240:354-368

Heidarvand L, Millar AH, Taylor NL (2017) Responses of the mitochondrial respiratory system to low temperature in plants. Crit Rev Plant Sci 36:217-240

Hoermiller Imke I, Naegele T, Augustin H, Stutz S, Weckwerth W, Heyer Arnd G (2017) Subcellular reprogramming of metabolism during cold acclimation in Arabidopsis thaliana. Plant Cell Environ 40:602-610

Kidokoro S, Yoneda K, Takasaki H, Takahashi F, Shinozaki K, Yamaguchi-Shinozaki K (2017) Different cold-signaling pathways function in the responses to rapid and gradual decreases in temperature. Plant Cell 29:760-774

Kirst M, Myburg AA, De León JPG, Kirst ME, Scott J, Sederoff R (2004) Coordinated genetic regulation of growth and lignin revealed by quantitative trait locus analysis of cDNA microarray data in an interspecific backcross of eucalyptus. Plant Physiol 135:2368-2378

Kooke R, Keurentjes JJB (2011) Multi-dimensional regulation of metabolic networks shaping plant development and performance. J Exp Bot 63:3353

Lisec J, Schauer N, Kopka J, Willmitzer L, Fernie AR (2006) Gas chromatography mass spectrometry-based metabolite profiling in plants. Nat Protoc 1:387-396

Livak KJ, Schmittgen TD (2001) Analysis of relative gene expression data using real-time quantitative PCR and the $2^{-\triangle \Delta C T}$ method. Methods 25:402-408

Lunn JE, Delorge I, Figueroa CM, Van Dijck P, Stitt M (2014) Trehalose metabolism in plants. Plant J 79:544-567

Mizrachi E, Hefer C, Ranik M, Joubert F, Myburg A (2010) De novo assembled expressed gene catalog of a fast-growing Eucalyptus tree produced by Illumina mRNA-Seq. BMC Genomics 11:681

Mokochinski JB, Mazzafera P, Sawaya ACHF, Mumm R, de Vos RCH, Hall RD (2018) Metabolic responses of Eucalyptus species to different temperature regimes. J Integr Plant Biol 60:397-411

Mora F, Serra N (2014) Bayesian estimation of genetic parameters for growth, stem straightness, and survival in Eucalyptus globulus on an Andean Foothill site. Tree Genet Genomes 10:711-719

Moura JCMS, Bonine CAV, De Oliveira Fernandes J, Viana MC Dornelas, Mazzafera P (2010) Abiotic and biotic stresses and changes in the lignin content and composition in plants. J Integr Plant Biol 52:360-376

Moura JCMS, Araújo P, Brito MS, Souza UR, Viana JOF, Mazzafera P (2012) Validation of reference genes from
Eucalyptus spp. under different stress conditions. BMC Res Notes 5:1-10

Navarrete-Campos D, Bravo L, Rubilar R, Emhart V, Sanhueza R (2013) Drought effects on water use efficiency, freezing tolerance and survival of Eucalyptus globulus and Eucalyptus globulus $\times$ nitens cuttings. New For 44:119-134

O’Brien MJ, Leuzinger S, Philipson CD, Tay J, Hector A (2014) Drought survival of tropical tree seedlings enhanced by non-structural carbohydrate levels. Nat Clim Change 4:710

Osakabe Y, Kajita S, Osakabe K (2011) Genetic engineering of woody plants: current and future targets in a stressful environment. Physiol Plant 142:105-117

Osakabe Y, Yamaguchi-Shinozaki K, Shinozaki K, Tran L-SP (2013) Sensing the environment: key roles of membranelocalized kinases in plant perception and response to abiotic stress. J Exp Bot 64:445-458

Quang T, Hallingbäck H, Gyllenstrand N, von Arnold S, Clapham D (2012) Expression of genes of cellulose and lignin synthesis in Eucalyptus urophylla and its relation to some economic traits. Trees 26:893-901

Radchuk R, Emery RJN, Weier D, Vigeolas H, Geigenberger P, Lunn JE, Feil R, Weschke W, Weber H (2010) Sucrose non-fermenting kinase 1 (SnRK1) coordinates metabolic and hormonal signals during pea cotyledon growth and differentiation. Plant J 61:324-338

Ruedell C, de Almeida M, Schwambach J, Posenato C, FettNeto A (2013) Pre and post-severance effects of light quality on carbohydrate dynamics and microcutting adventitious rooting of two Eucalyptus species of contrasting recalcitrance. Plant Growth Regul 69:235-245

Ruprecht C, Persson S (2012) Co-expression of cell wall related genes: new tools and insights. Front Plant Sci 3:83

Ruprecht C, Mutwil M, Saxe F, Eder M, Nikoloski Z, Persson S (2011) Large-scale co-expression approach to dissect secondary cell wall formation across plant species. Front Plant Sci 2:23

Saitou N, Nei M (1987) The neighbor-joining method: a new method for reconstructing phylogenetic trees. Mol Biol Evol 4:406-425

Schluepmann H, Berke L, Sanchez-Perez GF (2011) Metabolism control over growth: a case for trehalose-6-phosphate in plants. J Exp Bot 63:3379

Shin J, Sánchez-Villarreal A, Davis AM, Du S-X, Berendzen KW, Koncz C, Ding Z, Li C, Davis SJ (2017) The metabolic sensor AKIN10 medulates the Arabidopsis circadian clock in a light-dependent manner. Plant Cell Environ 40:997-1008

Smeekens S, Ma J, Hanson J, Rolland F (2010) Sugar signals and molecular networks controlling plant growth. Curr Opin Plant Biol 13:273-278

Tamura K, Peterson D, Peterson N, Stecher G, Nei M, Kumar S (2011) MEGA5: molecular evolutionary genetics analysis using maximum likelihood, evolutionary distance, and maximum parsimony methods. Mol Biol Evol 28:2731-2739

Tarkowski LP, Van den Ende W (2015) Cold tolerance triggered by soluble sugars: a multifaceted countermeasure. Front Plant Sci 6:203

Thompson JD, Higgins DG, Gibson TJ (1994) CLUSTAL W: improving the sensitivity of progressive multiple sequence alignment through sequence weighting, positions-specific 
gap penalties and weight matrix choice. Nucleic Acids Res 22:4673-4680

Tomé FS, Nägele T, Adamo M, Garg A, Marco-llorca C, Nukarinen E, Lorenzo P, Peviani A, Simeunovic A, Tatkiewicz A, Tomar M, Gamm M (2014) The low energy signaling network. Front Plant Sci 5:353

Turnbull JW, Booth TH (2002) Eucalyptus: the genus Eucalyptus. Taylor \& Francis, London, p 450

Warren CR (2011) How does P affect photosynthesis and metabolite profiles of Eucalyptus globulus? Tree Physiol 31:727-739

Warren C, Aranda I, Cano FJ (2012) Metabolomics demonstrates divergent responses of two Eucalyptus species to water stress. Metabolomics 8:186-200

Williams SP, Rangarajan P, Donahue JL, Hess JE, Gillaspy GE (2014) Regulation of sucrose non-fermenting related kinase 1 genes in Arabidopsis thaliana. Front Plant Sci $5: 324$
Wu L, Birch RG (2010) Physiological basis for enhanced sucrose accumulation in an engineered sugarcane cell line. Funct Plant Biol 37:1161-1174

Wurzinger B, Nukarinen E, Nägele T, Weckwerth W, Teige M (2018) The SnRK1 kinase as central mediator of energy signaling between different organelles. Plant Physiol 176:1085-1094

Zarka DG, Vogel JT, Cook D, Thomashow MF (2003) Cold induction of Arabidopsis CBF genes involves multiple ICE (Inducer of CBF Expression) promoter elements and a cold-regulatory circuit that is desensitized by low temperature. Plant Physiol 133:911-918

Publisher's Note Springer Nature remains neutral with regard to jurisdictional claims in published maps and institutional affiliations. 